\title{
Split-type free-displacer Stirling refrigerator design using linear network analysis
}

\author{
B.J. Huang and C.W. Lu
}

Department of Mechanical Engineering, National Taiwan University, Taipei 10617, Taiwan

Received 9 April 1996; revised 13 May 1996

A linear network analysis is developed for the system design of split-type free-displacer Stirling refrigerators. The dynamics models are derived to describe the input/output relation of each component by use of the governing equations in conjunction with linearization and approximation. By connecting the equivalent circuits of the components together, a linear network consisting of a fluid network and a displacer network is obtained. The fluid network accounts for the propagation of gas flow induced by the pressure wave; while the displacer network accounts for the displacer motion induced by the pressure force exerted on the displacer. Two transfer functions are further derived for the displacer motion and the gas pressure of the expansion space with respect to the piston motion. The system performance is then evaluated from the frequency response of the transfer functions by using the sinusoidal signal analysis. The performance prediction of a split-type free-displacer Stirling refrigerator using the linear network analysis is shown to be satisfactory. (C) 1996 Elsevier Science Limited

Keywords: Stirling refrigerator; split type Stirling refrigerator; linear network model of Stirling cooler

\begin{tabular}{|llll|}
\hline \multicolumn{2}{|l|}{ Nomenclature } & $V$ & volume $\left(\mathrm{m}^{3}\right)$ \\
$a$ & gain & $X$ & distance $(\mathrm{m})$ \\
$A$ & area $\left(\mathrm{m}^{2}\right)$ & $X$ & displacement $(\mathrm{m})$ \\
$C$ & flow capacitance $\left(\mathrm{m}^{3} \mathrm{~kg} / \mathrm{kJ}\right)$ & $\tau$ & time constant $(\mathrm{s})$ \\
$C_{\mathrm{d}}$ & displacer loss coefficient $(\mathrm{N}$ s $/ \mathrm{m})$ & $\omega$ & angular frequency $(=2 \pi f)$ \\
$C_{\mathrm{p}}$ & gas specific heat at constant pressure & $\rho$ & density \\
& (kJ/kg K) & $\theta$ & piston angle $(\mathrm{deg})$ \\
$C_{\mathrm{v}}$ & gas specific heat at constant volume $(\mathrm{kJ} / \mathrm{kg} \mathrm{K})$ & & \\
$h$ & convection heat transfer coefficient $\left(\mathrm{W} / \mathrm{m}^{2} \mathrm{~K}\right)$ & $S$ Subscripts \\
$d$ & diameter $(\mathrm{m})$ & $\mathrm{c}$ & compression chamber; characteristic \\
$f$ & frequency $(\mathrm{Hz})$ & & impedance \\
$k$ & thermal conductivity $(\mathrm{W} / \mathrm{m} \mathrm{K})$ & $\mathrm{d}$ & displacer \\
$K_{\mathrm{d}}$ & gas spring constant $(\mathrm{N} / \mathrm{m})$ & $\mathrm{e}$ & expansion space \\
$L$ & lenglh $(\mathrm{III})$ & $\mathrm{f}$ & gas; fluid; flow \\
$\dot{m}$ & mass flow rate $(\mathrm{kg} / \mathrm{s})$ & $\mathrm{g}$ & gas \\
$R$ & gas constant $(\mathrm{kJ} /(\mathrm{kgK}))$ & $\mathrm{h}$ & hot end; hydraulic \\
$R e$ & Reynolds number, dimensionless & $\mathrm{H}$ & hot end of the regenerator \\
$p$ & pressure $\left(\mathrm{N} / \mathrm{m}^{2}\right)$ & $\mathrm{i}$ & inlet \\
$P$ & pressure $\left(\mathrm{N} / \mathrm{m}^{2}\right)$ & $\mathrm{L}$ & cold end \\
$p_{\mathrm{ch}}$ & charge pressure $\left(\mathrm{N} / \mathrm{m}^{2}\right)$ & $\mathrm{m}$ & mean \\
$Q_{\mathrm{nct}}$ & net cooling capacity $(\mathrm{W})$ & $\mathrm{n}$ & natural frequency \\
$s$ & Laplace variable, complex & $\mathrm{o}$ & outlet; equilibrium point; amplitude \\
$T$ & temperature $(\mathrm{K})$ & $\mathrm{p}$ & piston \\
$t$ & time $(\mathrm{s})$ & $\mathrm{r}$ & regenerator \\
& & &
\end{tabular}


s solid material

$t \quad$ connecting tube

w warm space

\section{Superscripts}

steady-state point; equilibrium point perturbed value

time change rate
The thermal performance analysis of a Stirling refrigerator is very important at the hardware design stage. Researchers have attempted to develop an analytical method and a computer-aided tool for the design application. But the achievement is limited so far. The designer still relies heavily on personnel experience or trial and error. For a split-type freedisplacer Stirling refrigerator, the losses of wall friction and clearance seal leakage of the displacer are rarely predicted accurately. Hence, the analysis can deviate greatly from the test results.

Nodal analysis' is a typical method developed for freedisplacer Stirling refrigerator simulation. However, the time-variant conservation equations of mass, momentum and energy have to be solved at each node of the whole refrigerator until the solution converges at each time instant. The computation is then repeated for different designs or operating conditions in order to obtain a performance map of the refrigerator. The solution procedure is so complicated and time consuming that it usually requires use of a super-computer. In order to simplify the analysis and increase the computational speed, a linear network analysis is developed in the present study.

A split-type frec-displacer Stirling refrigerator comprises a compressor and a cold finger connected by a connecting tube. The cold finger contains a regenerator/displacer, a gas spring, and an expansion space (cold head) as shown in Figure 1. The displacer is driven by the gaseous pressure wave resulting from the reciprocating motion of the piston. The whole refrigerator thus operates at a cyclically steady state very close to sinusoidal. The refrigerator can thus be treated as a dynamical system with the piston displacement as the system input and the displacer displacement and the expansion space pressure as the system output ${ }^{2}$.

From the viewpoint of system dynamics, any component of a dynamical system is triggered by an input (physical force) and induces an output (dynamic response). A dynamics model can be derived to describe the input/output relation of each component by use of the governing equations in conjunction with linearization and approximations. A network model can be further derived from the electric circuit analogy. An equivalent circuit or system block diagram can then be used to represent the dynamic behaviour of each component. Connecting the analogous circuits of all the components together will lead to a linear network of the refrigerator ${ }^{3}$. For the split-type free-displacer Stirling refrigerator, a transfer-function model representing the performance of the refrigerator can be derived, and from this the system performance can be easily evaluated. A brief summary of the analysis has been published in the previous technical note ${ }^{2}$. A detailed description of the linear network analysis for the split-type free-displacer Stirling refrigerator is reported in the present paper.

\section{Dynamics model of components}

The split-type free-displacer Stirling refrigerator essentially consists of six components: namcly, compressor, connecting tube, warm space, regenerator, displacer, and expansion space. The dynamics model of each component can be derived. For simplification, the ideal gas assumption is used throughout the derivation.

\section{Compressor}

The compressor of a Stirling refrigerator consists of a compression space and a piston driven by a crank mechanism. Since the piston motion is very fast and the mixing in the compression chamber is severe, the gas temperature and pressure inside the compression chamber are assumed to

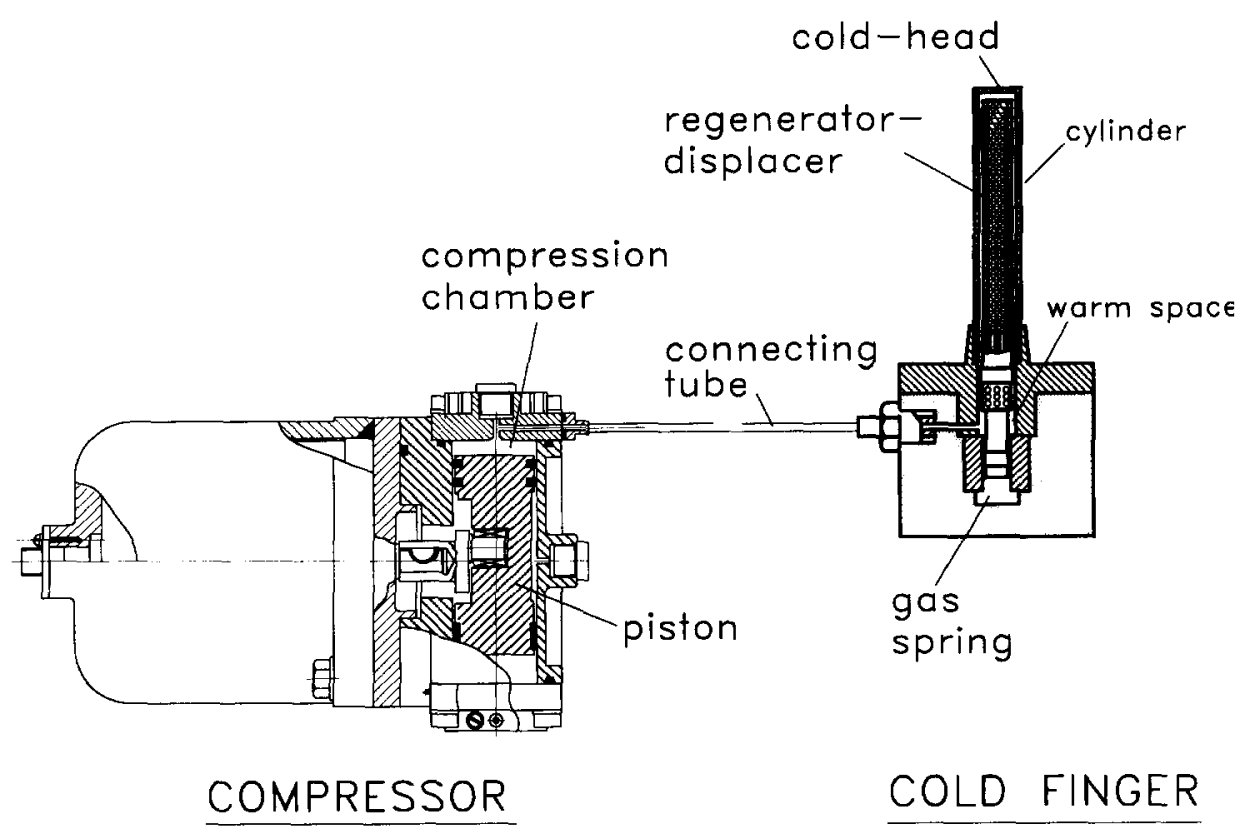

Figure 1 Schematic diagram of split-type free-displacer Stirling refrigerator 
be uniform. The actual gas compression process inside the compression chamber lies between isothermal and adiabatic. Since cooling is provided for the compressor of a Stirling refrigerator, the isothermal compression with a constant gas temperature $T_{\mathrm{c}}$ is assumed. Assuming no gas leakage between the piston and the cylinder, conservation of mass to the compression chamber will lead to the dynamics model

$$
\dot{m}_{\mathrm{c}}(t)=-\frac{1}{R T_{c}}\left\{V_{\mathrm{co}} \frac{d p_{\mathrm{c}}(t)}{d t}-A_{\mathrm{p}} p_{\mathrm{c}}(\mathrm{t}) \frac{\mathrm{d} X_{\mathrm{p}}(t)}{d t}\right\}
$$

$\dot{m}_{\mathrm{c}}$ is the mass flow rate out of the compressor; $p_{\mathrm{c}}$ is the gas pressure; $V_{c}(t)=V_{\text {co }}-A_{\mathrm{p}} X_{\mathrm{p}}(t) ; V_{\mathrm{co}}$ is the volume of compression chamber for the piston at the equilibrium position $\left(\bar{X}_{\mathrm{p}}=0\right) ; X_{\mathrm{p}}(t)$ is the piston displacement measured from the midpoint of the piston motion toward the top dead end; $A_{\mathrm{p}}$ is the cross section area of the piston.

Applying small perturbation around the equilibrium point, i.e. $X_{\mathrm{p}}(t)=\bar{X}_{\mathrm{p}}+\tilde{X}_{\mathrm{p}}(t) ; p_{\mathrm{c}}(t)=\bar{p}_{\mathrm{c}}+\bar{p}_{\mathrm{c}}(t) ; \dot{m}_{\mathrm{c}}(t)=$ $\tilde{\dot{m}}_{\mathrm{c}}+\tilde{\dot{m}}_{\mathrm{c}}(t)=\tilde{\dot{m}}_{\mathrm{c}}(t)$ to Equation (1), neglecting higher-order terms, taking that $\bar{X}_{\mathrm{p}}=0$ is the piston central position, and then taking Laplace transforms, we obtain a perturbed dynamics model

$$
\tilde{\dot{m}}_{\mathrm{p}}(s) \equiv \frac{s p_{o} A_{\mathrm{p}}}{R T_{\mathrm{c}}} \tilde{X}_{\mathrm{p}}(s)=\tilde{\dot{m}} c(s)+\frac{\tilde{p}_{\mathrm{c}}(s)}{1 / s C_{\mathrm{c}}}
$$

where $C_{\mathrm{c}}=V_{\mathrm{co}} / R T_{\mathrm{c}}$ is the equivalent capacitance of the compression chamber; $\widetilde{m}_{\mathrm{p}}(s)$ acts as a current source representing the available or gross mass flow generated by the piston motion. The equivalent circuit is shown in Figure 2.

\section{Connecting tube}

The connecting tube links the compressor and the cold finger. The flow field is assumed to be 1-D. In order for linearization, the second-order viscous and the inertia terms in the momentum equation are neglected. However, the second-order viscous friction is taken into account by a modified resistance coefficient $\kappa$ which is calculated using piecewise linear approximation ${ }^{4}$. Therefore, the viscous resistance is proportional to the mass flowrate with a proportional constant $\kappa$ depending upon the amplitude of the mass flow rate.

The gas in the connecting tube is assumed to undergo an isothermal process with a constant temperature $T_{\mathrm{t}}$. This can hold since the connecting tube is usually small in diameter and is thick in wall thickness in order to withstand the high gas pressure. Hence, the connecting tube can act as an energy storage medium to damp out the gas temperature

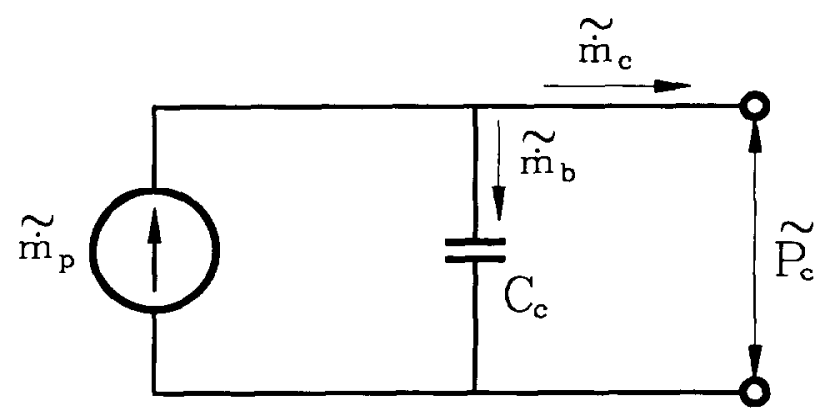

Figure 2 Equivalent circuit of compression chamber variation. From the above assumptions, we obtain the governing equations of the connecting tube from the conservations of mass and momentum.

$$
\begin{array}{r}
\frac{1}{R T_{\mathrm{t}}} \frac{\partial p(x, t)}{\partial t}+\frac{1}{A_{\mathrm{t}}} \frac{\partial \dot{\mathrm{m}}(x, t)}{\partial x}=0 \\
\frac{1}{A_{\mathrm{t}}} \frac{\partial \dot{\mathrm{m}}(x, t)}{\partial x}+\frac{\partial p(x, t)}{\partial x}+\frac{\kappa}{A_{\mathrm{t}}} \dot{m}(x, t)=0
\end{array}
$$

where $A_{\mathrm{t}}$ is the tube cross section area. It can be easily shown that Equations ( 3 ) and (4) are the wave equations.

Applying small perturbation around the equilibrium point and with $\overline{\dot{m}}=0$ for a cyclically-steady operation, i.e. $p(x, t)=\bar{p}+\tilde{p}(x, t) ; \tilde{m}(x, t)=\bar{m}+\tilde{\dot{m}}(x, t)=\tilde{m}(x, t)$, to Equations (3) and (4) and then solving the Laplace transformed equations, we obtain the dynamics model of the connecting tube

$$
\begin{aligned}
{\left[\begin{array}{c}
\tilde{m}_{\mathrm{to}}(s) \\
\tilde{p}_{\mathrm{to}}(s)
\end{array}\right] } & =\left[\begin{array}{cc}
\cosh \left(\Gamma_{\mathrm{t}} I_{\mathrm{t}}\right) & -\frac{1}{Z_{\mathrm{ct}}} \sinh \left(\Gamma_{\mathrm{t}} L_{\mathrm{t}}\right) \\
-Z_{\mathrm{ct}} \sinh \left(\Gamma_{\mathrm{t}} L_{\mathrm{t}}\right) & \cosh \left(\Gamma_{\mathrm{t}} L_{\mathrm{t}}\right)
\end{array}\right] \\
& \times\left[\begin{array}{c}
\tilde{\dot{m}}_{\mathrm{ti}}(s) \\
\tilde{p}_{\mathrm{ti}}(s)
\end{array}\right]
\end{aligned}
$$

where $\Gamma_{\mathrm{t}}=\sqrt{Z_{\mathrm{t}} Y_{\mathrm{t}}}$ and $Z_{\mathrm{ct}}=\sqrt{Z_{\mathrm{t}} / Y_{\mathrm{t}}}$ are the propagation constant and the characteristic impedance of the tube, respectivcly; $Z_{\mathrm{t}}=R_{\mathrm{Ft}}+s L_{\mathrm{Ft}}=$ series impedance and $Y_{\mathrm{t}}=s C_{\mathrm{Ft}}=$ shunt admittance; $\Gamma_{\mathrm{t}}$ and $Z_{\mathrm{ct}}$ are derived as

$$
\Gamma_{\mathrm{t}}=\sqrt{s C_{\mathrm{Ft}}\left(R_{\mathrm{Ft}}+s L_{\mathrm{Ft}}\right)} ; Z_{\mathrm{ct}}=\frac{\Gamma_{\mathrm{t}}}{s C_{\mathrm{Ft}}}
$$

where $C_{\mathrm{Ft}}, L_{\mathrm{Ft}}$ and $R_{\mathrm{Ft}}$ are the flow capacitance, flow inductance, and flow resistance per unit tube length, respectively, which are defined as

$$
C_{\mathrm{Ft}}=\frac{A_{\mathrm{t}}}{R T_{\mathrm{t}}} ; L_{\mathrm{Ft}}=\frac{1}{A_{\mathrm{t}}} ; R_{\mathrm{Ft}}=\frac{\kappa}{A_{\mathrm{t}}}
$$

where

$$
\kappa=0.1556\left(\rho_{\mathrm{t}} w_{\max } d_{\mathrm{r}} / \mu\right)^{-0.201}\left(w_{\max } / d_{\mathrm{h}}\right),
$$

$w_{\max }$ is the peak velocity of the oscillating flow in the tube; $d_{\mathrm{t}}$ is the hydraulic diameter of the tube ${ }^{\mathrm{s}}$.

It is worth noting that $\kappa$ is considered to be a constant during the modelling, but should be adjusted by numerical iteration during the computation to give a correct value for the corresponding mass flow $w_{\max }$.

Since the dynamics model of the connecting tube belongs to a distributed-parameter system, an equivalent circuit consisting of infinite sets of shunt and series impedances can be drawn as shown in Figure 3. Figure 3 approximates the model of Equation (5) as $N$ is large. The shunt and series impedances for each segment of the tube satisfy the following relation 


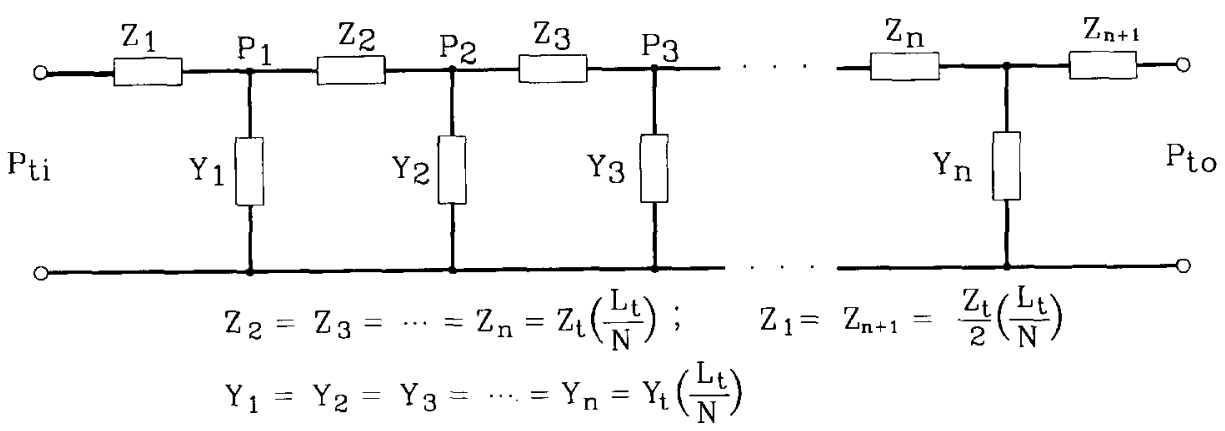

Figure 3 Equivalent circuit of connecting tube

$$
\begin{aligned}
& Z_{2}=Z_{3}=\cdots=Z_{\mathrm{n}}=Z_{\mathrm{t}}\left(\frac{L_{\mathrm{t}}}{N}\right) ; \\
& Z_{1}=Z_{\mathrm{n}+1}=\frac{Z_{\mathrm{t}}}{2}\left(\frac{L_{\mathrm{t}}}{N}\right) \\
& Y_{1}=Y_{2}=Y_{3}=\cdots=Y_{n}=Y_{\mathrm{t}}\left(\frac{L_{\mathrm{t}}}{N}\right)
\end{aligned}
$$

The limiting case $N \rightarrow \infty$ corresponds to the present model, Equation (5). A similar circuit can also be drawn based on the series expansion of $\cosh \left(\Gamma_{t} L_{t}\right)$ and $\sinh \left(\Gamma_{t} L_{t}\right)$ in Equation (5) with respect to $\Gamma_{\mathrm{t}} L_{\mathrm{t}}$.

A system block diagram as shown in Figure 4 is used here to illustrate the input/output relation of the connecting tube from the system dynamics point of view. In practice, Figure 3 will be used as the analogous circuit during the linear network analysis.

From Equation (5), we also obtain the relation between the output impedance $Z_{\mathrm{ta}}\left[\equiv \tilde{p}_{\mathrm{ta}}(s) / \tilde{m}_{\mathrm{ta}}(\mathrm{s})\right]$ and the input impedance $Z_{\mathrm{ti}}\left[\equiv \tilde{p}_{\mathrm{ti}}(s) / \dot{m}_{\mathrm{ti}}(s)\right]$ of the connecting tube

$$
Z_{\mathrm{ti}}=\frac{1+\frac{Z_{\mathrm{ct}}}{Z_{\mathrm{to}}} \tanh \left[\Gamma_{\mathrm{t}} L_{\mathrm{t}}\right]}{1+\frac{Z_{\mathrm{to}}}{Z_{\mathrm{ct}}} \tanh \left[\Gamma_{\mathrm{t}}(s) L_{\mathrm{t}}\right]}-Z_{\mathrm{to}}
$$

\section{Warm space}

The gas temperature is assumed uniform in the warm space since the gas mixing is severe. Similar to the compression chamber, a linearly perturbed model can be derived from mass continuity equation

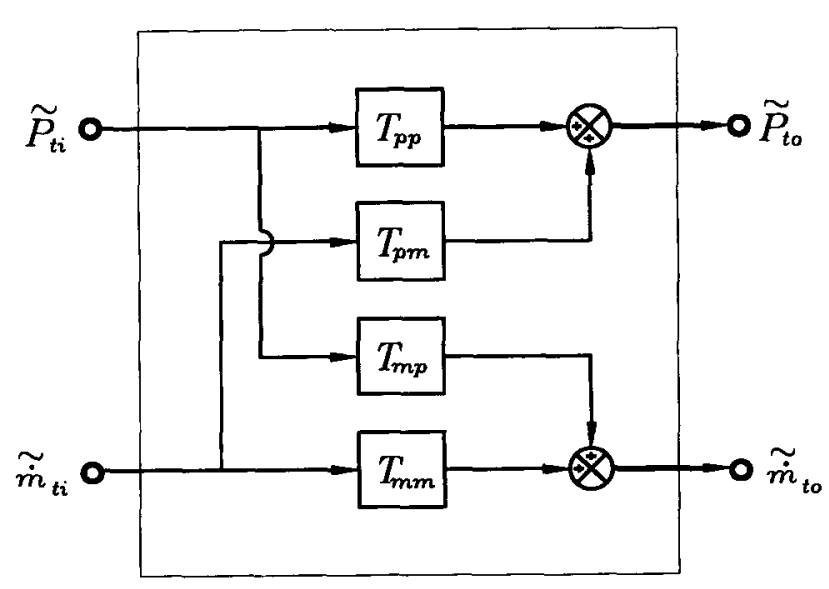

Figure 4 Block diagram of connccting tubc

$$
\tilde{\dot{m}}_{\mathrm{wi}}(s)=\tilde{\dot{m}}_{\mathrm{wo}}(s)+\frac{s p_{\mathrm{o}} A_{\mathrm{dw}}}{R T_{\mathrm{w}}} \tilde{X}_{\mathrm{d}}(s)+\frac{\tilde{p}_{\mathrm{w}}(s)}{1 / s C_{\mathrm{w}}}
$$

The second term in the right-hand side of Fquation (11) is the mass flow rate induced by the displacer motion, which is analogous to electric current source; the third term represents the flow capacitance effect of the warm space; $C_{w}$ $\equiv V_{\mathrm{wo}} /\left(R T_{\mathrm{w}}\right)$. The equivalent electric circuit is shown in Figure $S$.

\section{Regenerator}

The regenerator is an energy-storage element made from wire screens. The derivation of the dynamical model is similar to that of the connecting tube.

For the momentum equation, the inertia term $\left(1 / A_{\mathrm{fr}}^{2}\right) \partial(\dot{m}|\dot{m}| / \rho) / \partial \mathrm{x}$ and the second-order viscous term $\rho \beta\left(\epsilon / \rho A_{\mathrm{fr}}\right)^{2} \dot{m}|\dot{m}|$ can be neglected. This can hold since the Reynolds number in the regenerator is not large. The pressure loss due to the second-order viscous friction is taken into account by a modified frictional coefficient $\bar{\alpha}$ calculated using piecewise linear approximation ${ }^{4}$. Therefore, the viscous resistance is assumed to be proportional to the mass flow with a proportional constant $\bar{\alpha}$ which depends upon the amplitude $\dot{m}_{\max }$ of the oscillating flow. $\bar{\alpha}$ is considered to be a constant during the modelling, however it should be adjusted by numerical iteration during the computation in order to give a correct value for the corresponding mass flow $\left(\dot{n}_{\max }\right)$.

Assuming 1-D flow, no axial conduction and constant properties, the transient governing equations in terms of perturbed variables $\dot{m}(x, t)=\tilde{\dot{m}}_{r}(x)+\tilde{\dot{m}}(x, t), p(x, t)=\bar{P}_{r}(x)+$ $\tilde{p}(x, t)$, and $T(x, t)=\bar{T}_{\mathrm{r}}(x)+\tilde{T}(x, t)$ and using $\tilde{\dot{m}}_{\mathrm{r}}(x)=0$ for cyclically-steady operation, are derived from the conservation of mass and momentum

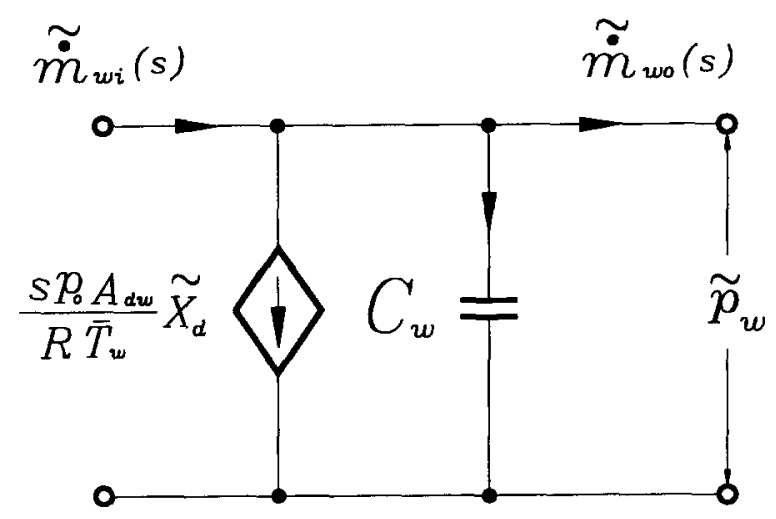

Figure 5 Equivalent circuit of warm space 


\section{Continuity equation of gas}

$$
\begin{gathered}
\bar{T}_{\mathrm{r}}(x) \frac{\partial \tilde{P}(x, t)}{\partial t}-\bar{P}_{\mathrm{r}}(x) \frac{\partial \tilde{T}(x, t)}{\partial t} \\
+\frac{R \bar{T}_{\mathrm{r}}^{2}(x)}{A_{\mathrm{fr}}} \frac{\partial \tilde{\dot{m}}(x, t)}{\partial x}=0
\end{gathered}
$$

\section{Momentum equation of gas}

$$
L_{\mathrm{Fr}} \frac{\partial \tilde{\dot{m}}(x, t)}{\partial t}+\frac{\partial \tilde{p}(x, t)}{\partial x}+R_{\mathrm{Fr}} \tilde{\dot{m}}(x, t)=0
$$

where $R_{\Gamma \mathrm{r}}$ is the regenerator flow resistance per unit length; $L_{\mathrm{Fr}}$ is the flow inductance per unit length;

$$
R_{\mathrm{Fr}}=\frac{\bar{\alpha} \epsilon \nu}{A_{\mathrm{fr}}} ; L_{\mathrm{Fr}}=\frac{1}{A_{\mathrm{fr}}},
$$

where $\bar{\alpha}=\alpha+\left(\beta / d_{\mathrm{h}}\right) \epsilon R_{\mathrm{eh}} ; \alpha=175 /\left(2 \epsilon d_{\mathrm{h}}^{2}\right), \beta=1.6 /\left(2 \epsilon^{2} d_{\mathrm{h}}\right)$ can be obtained from Tanaka's results; $d_{\mathrm{h}}=$ hydraulic diameter $=\epsilon d_{\mathrm{m}} /(1-\epsilon) ; h=0.33\left(k_{\mathrm{f}} / d_{\mathrm{h}}\right) R_{\mathrm{eh}}^{0.67}$ based on Tanaka's results ${ }^{5} ; d_{\mathrm{m}}$ is the matrix wire diameter.

For simplification, the axial variation of gas temperature and pressure at steady state are approximated by the average values, i.e. $\bar{T}_{\mathrm{r}}(x) \approx T_{\mathrm{rm}}=\left(T_{\mathrm{H}}+T_{\mathrm{L}}\right) / 2$ and $\bar{P}_{\mathrm{r}}(x) \approx P_{\mathrm{rm}}$ $=P_{c h}$. This was justified experimentally, as the temperature distribution in the regenerator is roughly linear.

Equations (12) and (13) cannot be solved since the gas temperature $\tilde{T}(x, t)$ is not known. The following energy equations for the gas and the screen matrix are thus derived using the above approximation on $\bar{T}_{\mathrm{r}}(x)$ and $\bar{P}_{\mathrm{r}}(x)$

\section{Energy equation of gas}

$$
\begin{aligned}
& \frac{C_{\mathrm{Fr}}}{\gamma} \frac{\partial \tilde{p}(x, t)}{\partial t}+\frac{\partial \tilde{\dot{m}}(x, t)}{\partial x} \\
& +\frac{C_{\operatorname{Tr}}}{\gamma \tau_{\mathrm{gr}}}\left[\tilde{T}(x, t)-\tilde{T}_{\mathrm{s}}(x, t)\right]=0 .
\end{aligned}
$$

\section{Energy equation of regenerator matrix}

$$
\tau_{\mathrm{sr}} \frac{\partial \tilde{T}_{\mathrm{s}}(x, t)}{\partial t}+\left[\tilde{T}_{\mathrm{s}}(x, t)-\tilde{T}(x, t)\right]=0
$$

where $C_{\mathrm{Tr}}$ and $C_{\mathrm{Tr}}$ are the flow capacitance per unit length due to pressure change and temperature change, respectively; $\tau_{\mathrm{gr}}$ and $\tau_{\mathrm{sr}}$ are time constants of gas and matrix, respectively, and $\gamma=C_{\mathrm{p}} / C_{\mathrm{v}} ; x$ is the position measured from the hot side of the regenerator;

$$
\begin{aligned}
C_{\mathrm{Fr}} & =\frac{A_{\mathrm{fr}}}{R T_{\mathrm{rm}}} ; C_{\mathrm{Tr}}=\frac{P_{\mathrm{rm}} A_{\mathrm{fr}}}{R T_{\mathrm{rm}}^{2}} ; \\
\tau_{\mathrm{gr}} & =\frac{P_{\mathrm{rm}} \epsilon V_{\mathrm{o}} C_{\mathrm{v}}}{R T_{\mathrm{rm}} h A_{\mathrm{HT}}} ; \tau_{\mathrm{sr}}=\frac{\rho_{\mathrm{s}}(1-\epsilon) V_{\mathrm{o}} C_{\mathrm{s}}}{h A_{\mathrm{HT}}}
\end{aligned}
$$

where $A_{\mathrm{HT}}$ is the surface area of the regenerator $=4 V_{\mathrm{o}}(1-$ $\epsilon) / d_{\mathrm{m}} \cdot d_{\mathrm{m}}$ is the wire diameter of screen disks; $R_{\mathrm{eh}}$ is the Reynolds number based on $d_{\mathrm{h}} ; k_{\mathrm{f}}$ is the gas thermal conductivity.

Solution of Equations (12), (13), (15) and (16) can be obtained by Laplace transforms. Combining Equations (15) and (16) with (12) and (13), we obtain the gas continuity equation as

$$
\frac{d \tilde{\dot{m}}(x, s)}{d x}+s C_{\mathrm{FTr}} \tilde{p}(x, s)=0
$$

and the gas momentum equation as

$$
\frac{d \tilde{p}(x, s)}{d x}+\left(R_{\mathrm{Fr}}+s L_{\mathrm{F}}\right) \tilde{\dot{m}}(x, s)=0 .
$$

where $C_{\mathrm{FTr}}$ is the regenerator flow capacitance due to pressure change and time responses of gas and matrix that is derived as

$$
C_{\mathrm{FTr}}=C_{\mathrm{Fr}} \frac{1+\tau_{\mathrm{sr}} /\left[\tau_{\mathrm{gr}}\left(1+s \tau_{\mathrm{sr}}\right)\right]}{\gamma+\tau_{\mathrm{sr}} /\left[\tau_{\mathrm{gr}}\left(1+s \tau_{\mathrm{sr}}\right)\right]} .
$$

It is worth noting that Equations (18) and (19) are the wave equations.

A linearly-perturbed dynamics model for the regenerator can be obtained ${ }^{4}$

$$
\begin{aligned}
{\left[\begin{array}{c}
\tilde{\dot{m}}_{\mathrm{ro}}(s) \\
\tilde{p}_{\mathrm{ro}}(s)
\end{array}\right] } & =\left[\begin{array}{cc}
\cosh \left(\Gamma_{\mathrm{r}} L_{\mathrm{r}}\right) & -\frac{1}{Z_{\mathrm{cr}}} \sinh \left(\Gamma_{\mathrm{r}} L_{\mathrm{r}}\right) \\
-\mathrm{Z}_{\mathrm{cr}} \sinh \left(\Gamma_{\mathrm{r}} L_{\mathrm{r}}\right) & \cosh \left(\Gamma_{\mathrm{r}} L_{\mathrm{r}}\right)
\end{array}\right] \\
& \times\left[\begin{array}{c}
\tilde{m}_{\mathrm{ri}}(s) \\
\tilde{p}_{\mathrm{ri}}(s)
\end{array}\right]
\end{aligned}
$$

where $\Gamma_{\mathrm{r}}=\sqrt{Z_{\mathrm{r}} Y_{\mathrm{r}}}=$ regenerator propagation constant and $Z_{\mathrm{cr}}=\sqrt{Z_{\mathrm{r}} / \bar{Y}_{\mathrm{r}}}=$ regenerator characteristic impedance; they are derived as

$$
\begin{aligned}
\Gamma_{\mathrm{r}} & =\sqrt{s C_{\Gamma \mathrm{Tr}}\left(R_{\mathrm{Fr}}+s L_{\mathrm{Tr}}\right)} ; \\
Z_{\mathrm{cr}} & -\frac{\Gamma_{\mathrm{r}}}{s C_{\mathrm{FTr}}}
\end{aligned}
$$

Similar to the connecting tube, the dynamics model of the regenerator belongs to a distributed-parameter system. An equivalent circuit consisting of infinite sets of shunt and series impedances similar to Figure 3 can be drawn. This is based on the series expansion of $\cosh \Gamma_{\mathrm{r}} L_{\mathrm{r}}$ and $\sinh \Gamma_{\mathrm{r}} L_{\mathrm{r}}$ in Equation (21) with respect to $\Gamma_{\mathrm{r}} L_{\mathrm{r}}$. A system block diagram similar to Figure 4 can be used to represent the input/output relation of the regenerator.

Equation (21) represents the dynamics model of the regenerator in terms of the pressure and mass flowrate that are derived from the conservation equations of continuity and momentum. The conservation equation of encrgy can also be simultaneously solved using the solutions of $\tilde{p}_{\mathrm{r}}(x, s)$ and $\dot{m}_{\mathrm{r}}(\tilde{x}, s)$ to obtain the gas temperature in the regenerator $\widetilde{T}_{\text {fr }}(x, s)$. The gas temperature at the cold side of the regenerator is finally derived as

$$
\begin{aligned}
\tilde{T}_{\mathrm{fr}}\left(L_{\mathrm{r}}, s\right)= & -\frac{C_{\mathrm{Fr}}}{C_{\mathrm{Tr}}} \frac{\tau_{\mathrm{gr}}\left(1+s \tau_{\mathrm{sr}}\right)}{\tau_{\mathrm{sr}}} \tilde{p}_{\mathrm{ro}}(s) \\
& -\frac{\gamma}{C_{\mathrm{Tr}}} \frac{\tau_{\mathrm{gr}}\left(1+s \tau_{\mathrm{sr}}\right) \Gamma_{\mathrm{r}}}{s \tau_{\mathrm{sr}} \sinh \left[\Gamma_{\mathrm{r}} L_{\mathrm{r}}\right]} \\
& \left\{\tilde{\dot{m}}_{\mathrm{ro}}(s) \cosh \left[\Gamma_{\mathrm{r}} L_{\mathrm{r}}\right]-\tilde{m}_{\mathrm{ri}}(s)\right\} .
\end{aligned}
$$




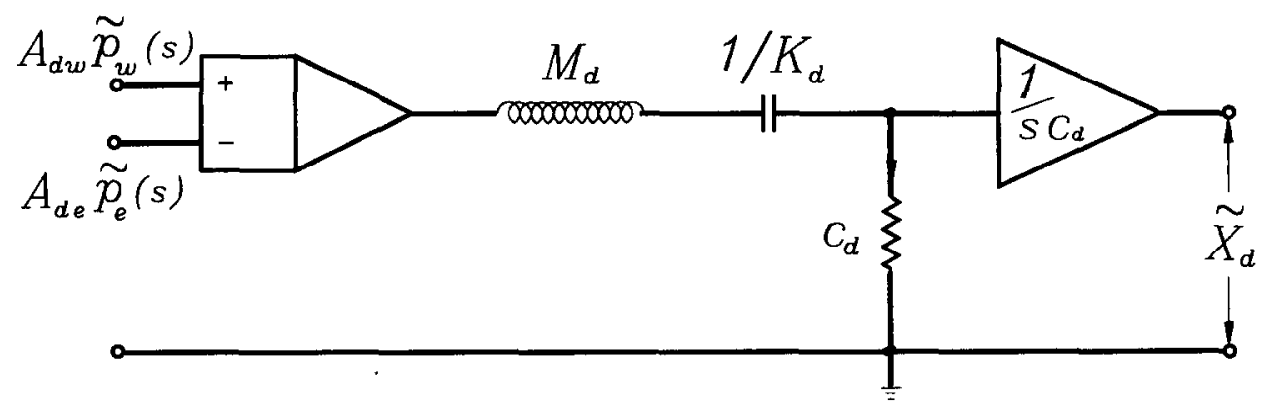

Figure 6 Equivalent circuit of displacer

\section{Displacer}

Taking force balance to the displacer will lead to a displacer dynamics model

$$
\begin{gathered}
\tilde{X}_{\mathrm{d}}(s)=\left[A_{\mathrm{dw}} \tilde{p}_{\mathrm{w}}(s)-A_{\mathrm{de}} \tilde{p}_{\mathrm{e}}(s)\right] \\
\left(\frac{1}{M_{\mathrm{d}} s^{2}+C_{\mathrm{d}} \mathrm{s}+K_{\mathrm{d}}}\right)
\end{gathered}
$$

where $K_{\mathrm{d}}$ is the spring constant, for gas spring at isothermal condition $K_{\mathrm{d}}=p_{\mathrm{o}} A_{\mathrm{dgs}}^{2} / V_{\mathrm{gs}} ; V_{\mathrm{gs}}=V_{\mathrm{ogs}}+A_{\mathrm{dgs}} \bar{X}_{\mathrm{d}} C_{\mathrm{d}}$ is the coefficient resulting from the friction and the leakage losses; $M_{\mathrm{d}}$ is the displacer mass. The equivalent circuit of the displacer is shown in Figure 6.

\section{Expansion space}

The cold space is usually made of a small empty space, sometimes filled with a porous medium to enhance the heat transfer between the gas and the wall. The gas enters the cold space with phase difference between the temperature and the mass flow. The gas agitation in the cold space is so severe that an uniform temperature with $T_{\text {ce }}$ in the cold space can be assumed. Similar to the warm space, a lumped model is derived from the mass conservation equation

$$
\tilde{\dot{m}}_{\mathrm{e}}(s)=\frac{\tilde{p}_{\mathrm{e}}(s)}{1 / s C_{\mathrm{e}}}-\frac{s p_{\mathrm{o}} A_{\mathrm{dc}}}{R T_{\mathrm{ce}}} \tilde{X}_{\mathrm{d}}(s)
$$

where $C_{\mathrm{e}} \equiv V_{\mathrm{eo}} /\left(R T_{\mathrm{ce}}\right)$ is the flow capacitance. The second term in the right-hand side of Equation (25) is the mass flowrate induced by the displacer motion, which is analogous to a current source. The equivalent circuit of the expansion space is shown in Figure 7.

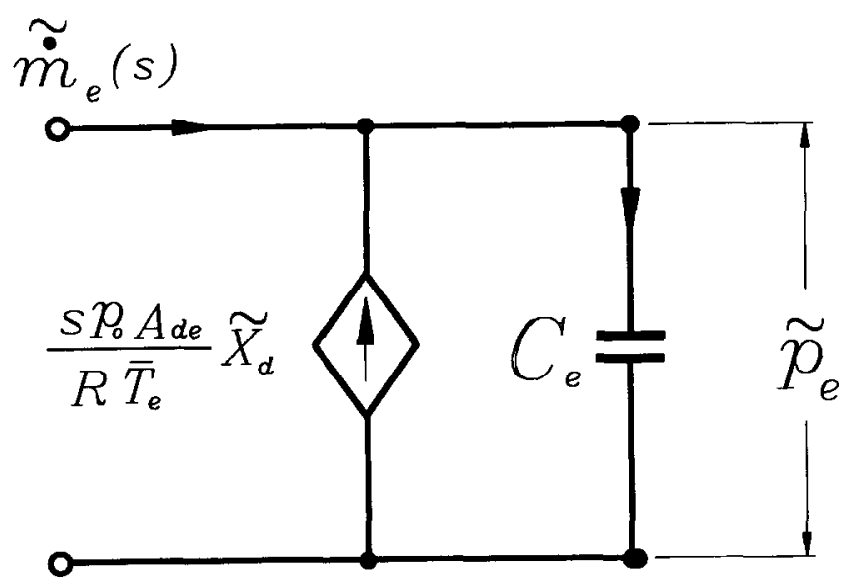

Figure 7 Equivalent circuit of expansion space

\section{Linear network of Stirling refrigerator}

Combining the equivalent circuits or block diagrams of all the components according to the process of the Stirling refrigerator, we obtain the linear network as shown in Figure 8 . The block diagrams for the connecting tube and the regenerator in Figure 8 imply the equivalent circuit of Figure 3.

It is worth noting that there are two networks for the split-type free-displacer Stirling refrigerator: namely, fluid network and displacer network. The lower part of Figure 8 is the fluid network with mass flow analogous to current and pressure analogous to voltage. The fluid network depicts the gaseous flow propagation from the compressor to the expansion space induced by the pressure.

The upper part of Figure 8 is the displacer network which picks up the pressure difference signal (analogue to voltage) across the warm space and the expansion space as the input and the displacer motion $X_{\mathrm{d}}(t)$ as the output. The displacer network thus depicts the displacer motion induced by the pressure force exerted on the displacer.

Physically, the fluid network and the displacer network are coupled since the gaseous mass flow, the pressure, and the displacer have mutually affected each other. The solutions of gas flow, pressure and displacer motion can be determined analytically from the network analysis.

\section{Transfer function of split-type free-displacer Stirling refrigerator}

A block diagram can be drawn as in Figure 8 for the connecting tube and regenerator with the distributed-parameter models. The transfer function model of the refrigerator cannot be derived directly from the circuit. Combining Equations (1) to (24) and rearranging these will lead to an equivalent impedance at the inlet of the connecting tube $Z_{1}$ :

$$
\begin{aligned}
Z_{\mathrm{ti}}(s) & =\frac{G_{8}(s)+G_{7}(s) Z_{\mathrm{ct}}(s) \tanh \left[\Gamma_{\mathrm{t}}(s) L_{\mathrm{t}}\right]}{G_{7}(s)+\left[G_{8}(s) / Z_{\mathrm{ct}}(s)\right] \tanh \left[\Gamma_{\mathrm{t}}(s) L_{\mathrm{t}}\right]} \\
G_{8}(s) & =R_{\mathrm{mm}}(s)\left[1+\frac{W_{\mathrm{md}}(s) G_{2}(s)}{1-G_{5}(s)}\right] \\
& -E_{\mathrm{me}}(s)\left[R_{\mathrm{pm}}(s)+\frac{G_{2}(s) G_{6}(s)}{1-G_{5}(s)}\right] \\
& -\frac{E_{\mathrm{md}}(s) G_{2}(s)}{1-G_{5}(s)} \\
G_{7}(s) \tilde{p}_{\mathrm{to}}(s) & =G_{8}(s) \tilde{\dot{m}}_{\mathrm{to}}(s) \\
G_{6}(s) & =R_{\mathrm{pm}}(s) W_{\mathrm{md}}(s)
\end{aligned}
$$




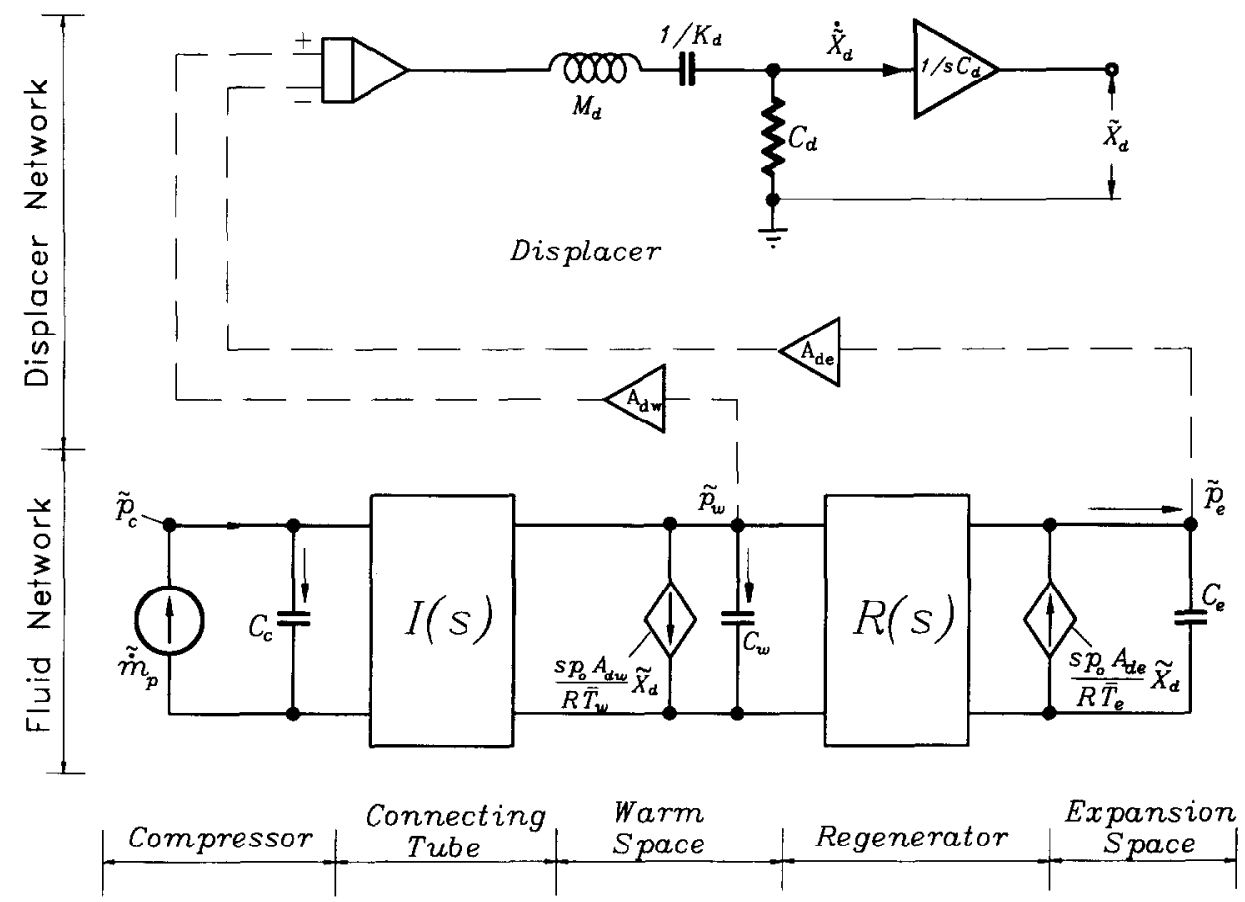

Figure 8 Linear network of split-type Stirling refrigerator

$$
\begin{aligned}
& G_{5}(s)= G_{2}(s) W_{\mathrm{md}}(s) \\
& G_{4}(s)= R_{\mathrm{pp}}(s)+R_{\mathrm{pm}}(s) W_{\mathrm{mp}}(s) \\
& G_{3}(s)= G_{1}(s)+G_{2}(s) W_{\mathrm{mpp}}(s) ; \\
& G_{2}(s)= D_{\mathrm{dc}}(s) R_{\mathrm{pm}}(s) \\
& G_{1}(s)= D_{\mathrm{dw}}(s)+D_{\mathrm{de}}(s) R_{\mathrm{pp}}(s) \\
& E_{\mathrm{me}}(s)= \frac{s V_{\mathrm{co}}}{R T_{\mathrm{ce}}} \\
& W_{\mathrm{mp}}(s)=-\frac{s V_{\mathrm{wo}}}{R T_{\mathrm{w}}} \\
& W_{\mathrm{md}}(s)=-\frac{s p_{\mathrm{v}} A_{\mathrm{dw}}}{R T_{\mathrm{w}}} \\
& R_{\mathrm{mm}}(s)=R_{\mathrm{pp}}(s)=\cosh \left(\Gamma_{\mathrm{r}} L_{\mathrm{r}}\right) ; \\
& R_{\mathrm{pm}}(s)=-Z_{\mathrm{cr}} \sinh \left(\Gamma_{\mathrm{r}} L_{\mathrm{r}}\right) \\
& D_{\mathrm{dc}}(s)=-\frac{A_{\mathrm{dc}}}{M_{\mathrm{d}} s^{2}+C_{\mathrm{d}} s+K_{\mathrm{d}}} ; \\
& D_{\mathrm{dw}}(s)=\frac{A_{\mathrm{dw}}}{M_{\mathrm{d}} s^{2}+C_{\mathrm{d}} s+K_{\mathrm{d}}}
\end{aligned}
$$

Therefore, the linear network model in Figure 8 can be transformed to Figure 9, which is the equivalent circuit at the inlet of the connecting tube.

The equivalent impedance at the current source $\tilde{\dot{m}}_{\mathrm{p}}$ is $Z_{\text {sys }}$ $(s)$ which follows

$$
1 / Z_{\mathrm{sys}}(s)=1 / Z_{\mathrm{c}}(s)+1 / Z_{\mathrm{ti}}(s)
$$

where $Z_{\mathrm{c}}(s)=1 / s C_{\mathrm{c}}=R T_{\mathrm{c}} / s V_{\mathrm{co}}$. Therefore, the flowrate at the connecting tube inlet is

$$
\tilde{\dot{m}}_{\mathrm{i}}(s)=\frac{s p_{\mathrm{o}} A_{\mathrm{p}} /\left(R T_{\mathrm{ce}}\right)}{1+Z_{\mathrm{ii}}(s) / Z_{\mathrm{c}}(s)} \tilde{X}_{\mathrm{p}}(s)
$$

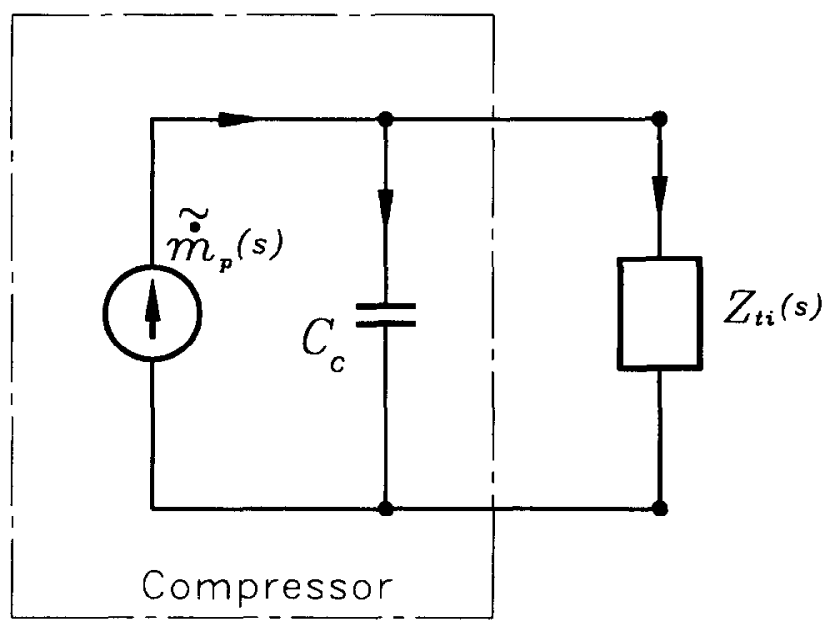

Figure 9 Equivalent circuit at connecting tube inlet

Substituting the above equations into the models of regenerator and connecting tube and rearranging, we obtain a relation for $\tilde{X}_{\mathrm{d}}(s)$ and $\tilde{X}_{\mathrm{p}}(s)$ and another relation for $\tilde{p}_{\mathrm{e}}(s)$ and $\tilde{X}_{\mathrm{p}}(s)$. Two system transfer functions of the split-type free-displacer Stirling refrigerator are thus obtained

$$
\begin{aligned}
G_{\mathrm{dp}}(s) \equiv & \frac{\tilde{X}_{\mathrm{d}}(s)}{\tilde{X}_{\mathrm{p}}(s)}= \\
& {\left[\frac{G_{3}(s) G_{8}(s) / G_{7}(s)+G_{2}(s)}{1-G_{5}(s)}\right] \times } \\
& {\left[\frac{I_{\mathrm{mp}}(s) Z_{\mathrm{ii}}(s)+I_{\mathrm{mm}}(s)}{1+Z_{\mathrm{ti}}(s) / Z_{\mathrm{c}}(s)}\right] \times } \\
& \frac{s p_{\mathrm{o}} A_{\mathrm{p}}}{R T_{\mathrm{c}}}
\end{aligned}
$$




$$
\begin{aligned}
G_{\mathrm{ep}}(s) \equiv & \frac{\tilde{p}_{\mathrm{c}}(s)}{\tilde{X}_{\mathrm{p}}(s)} \\
= & \left\{\left[G_{4}(s)+\frac{G_{3}(s) G_{6}(s)}{1-G_{5}(s)}\right] \frac{G_{8}(s)}{G_{7}(s)}\right. \\
& \left.+\left[R_{\mathrm{pm}}(s)+\frac{G_{2}(s) G_{6}(s)}{1-G_{5}(s)}\right]\right\} \\
& \times\left[\frac{I_{\mathrm{mp}}(s) Z_{\mathrm{ti}}(s)+I_{\mathrm{mm}}(s)}{1+Z_{\mathrm{ti}}(s) / Z_{\mathrm{c}}(s)}\right] \times \frac{s p_{0} A_{\mathrm{p}}}{R T_{c}}
\end{aligned}
$$

where

$$
I_{\mathrm{mm}}(s)=\cosh \left(\Gamma_{\mathrm{t}} L_{\mathrm{t}}\right) ; I_{\mathrm{mp}}(s)=-\frac{\sinh \left(\Gamma_{\mathrm{t}} L_{\mathrm{t}}\right)}{Z_{\mathrm{ct}}}
$$

It is seen that the system input of the split-type freedisplacer Stirling refrigerator is the piston displacement $X_{\mathrm{p}}$ of the compressor. There are two system outputs: displacer displacement $X_{\mathrm{d}}$ and expansion space pressure $p_{\mathrm{c}}$. The splittype Stirling refrigerator thus belongs to a single-input-minltiple-output (SIMO) dynamical system.

\section{Performance calculation}

\section{Maximum available cooling capacity}

The maximum available cooling capacity of a free-displacer Stirling refrigerator can be evaluated by integrating the expansion space pressure $p_{\mathrm{e}}(t)$ and volume $V_{\mathrm{e}}(t)$, where $V_{\mathrm{e}}(t)$ is related to the displacer displacement $X_{\mathrm{d}}(t)$. For a given piston motion $X_{\mathrm{p}}(t), X_{\mathrm{d}}(t)$ and $p_{\mathrm{e}}(t)$ can be obtained from the inverse of the system transfer functions $G_{\mathrm{dp}}(s)$ and $G_{\text {ep }}(s)$, Equations (38) and (39). However, this computation procedure is quite complicated. A simplification is needed. Since the piston is driven by a crank-shaft mechanism, the piston and the displacer motion as well as the generated pressure and mass flow waves inside the refrigerator approach sinusoidal. $X_{\mathrm{d}}(t)$ and $p_{\mathrm{e}}(t)$ can thus be computed simply from the gain and the phase of the frequency response functions, $G_{\mathrm{dp}}(j \omega)$ and $G_{\mathrm{ep}}(j \omega)$.

Assuming that $a_{\mathrm{dp}}$ is the gain of $G_{\mathrm{dp}}(j \omega)$ with phase $\phi_{\mathrm{d}}$ leading the piston; $a_{\mathrm{ep}}$ is the gain of $G_{\mathrm{ep}}(j \omega)$ with phase $\phi_{\mathrm{c}}$ leading the piston. Then

$$
\begin{aligned}
\tilde{p}_{\mathrm{e}}(t) & =p_{\mathrm{eo}} \sin \left(\omega t+\phi_{\mathrm{e}}\right) \\
& =p_{\mathrm{co}} \sin \left(\theta+\phi_{\mathrm{e}}\right)=\tilde{p}_{\mathrm{e}}(\theta) \\
\tilde{X}_{\mathrm{d}}(t) & =X_{\mathrm{do}} \sin \left(\omega t+\phi_{\mathrm{d}}\right) \\
& =X_{\mathrm{do}} \sin \left(\theta+\phi_{\mathrm{d}}\right)=\tilde{X}_{\mathrm{d}}(\theta)
\end{aligned}
$$

where $\theta$ is the piston angle; $p_{\text {eo }}$ and $X_{\mathrm{do}}$ are the amplitudes of $\tilde{p}_{\mathrm{c}}(t)$ and $\tilde{X}_{\mathrm{d}}(t)$, respectively. The maximum available cooling capacity of the Stirling refrigerator is

$$
\begin{aligned}
Q_{\max } & =f \oint_{0}^{2 \pi} p_{\mathrm{e}} d V_{\mathrm{e}} \\
& =f \pi a_{\mathrm{ep}} a_{\mathrm{dp}} X_{\mathrm{po}}^{2} A_{\mathrm{de}} \sin \left(\phi_{\mathrm{d}}-\phi_{\mathrm{e}}\right)
\end{aligned}
$$

where $X_{\mathrm{po}}$ is the amplitude of the piston, $f$ is the operating frequency.

\section{Net cooling capacity}

The net cooling capacity $Q_{\text {net }}$ can be evaluated by subtracting the heat losses from the maximum available cooling capacity $Q_{\max }$. There are four types of heat loss: heat conduction loss of regenerator $Q_{\text {cond }}$, enthalpy flow loss of regenerator $Q_{\text {enth }}$, shuttle heat loss of displacer $Q_{\text {shutle, }}$ and hysteresis loss of gas spring $W_{\mathrm{ir}}$, where

$$
Q_{\text {shu }}=k_{\mathrm{f}} \pi D_{\mathrm{c}} S_{\mathrm{e}}^{2} C_{\mathrm{T}} \frac{T_{\mathrm{H}}-T_{\mathrm{L}}}{2 c I_{\mathrm{r}}}
$$

where $D_{\mathrm{c}}$ is the outside diameter of displacer; $S_{\mathrm{c}}$ is the displacer stroke; $C_{\mathrm{T}}$ is the time fraction of heat transfer; $c$ is the clearance between displacer and cylinder; $L_{\mathrm{r}}$ is regenerator length. Usually, $C_{\mathrm{T}}=0.2$. The regenerator conduction loss is

$$
Q_{\text {cond }}=\mathrm{k}_{\mathrm{eff}}\left(\pi D_{\mathrm{e}}^{2} / 4\right) \frac{T_{\mathrm{H}}-T_{\mathrm{L}}}{L_{\mathrm{r}}}
$$

$k_{\mathrm{eff}}$ is the effective themmal conductivity of the regenerator. The enthalpy loss of the regenerator can be evaluated by integrating the mass flow rate and temperature waves at the boundary between the expansion space and the regenerator

$$
\begin{aligned}
Q_{\text {enth }} & =f \oint \dot{m}_{\text {ro }}(t) C_{\mathrm{p}} T_{\mathrm{f}}\left(L_{\mathrm{r}}, t\right) d t \\
& =f \pi C_{\mathrm{p}}\left|\tilde{\dot{m}}_{\mathrm{ro}}\right|\left|\tilde{T}_{\mathrm{f}}\left(L_{\mathrm{r}}\right)\right| \cos \left(\phi_{\mathrm{m}}-\phi_{\mathrm{T}}\right)
\end{aligned}
$$

$\phi_{\mathrm{m}}$ and $\phi_{\mathrm{T}}$ are, respectively, the phases of $\dot{m}_{\mathrm{ro}}(t)$ and $T_{\mathrm{f}}\left(L_{\mathrm{r}}, t\right)$ leading the piston. The hysteresis loss of gas spring can be evaluated by the following relation ${ }^{6}$

$$
W_{\mathrm{ir}}=\frac{k}{4} \sqrt{\frac{\omega \rho C_{\mathrm{p}}}{k}} \gamma(\gamma-1) T_{\mathrm{w}} A_{\mathrm{dw}}\left(\Delta V_{\mathrm{ogs}} / V_{\mathrm{ogs}}\right)^{2}
$$

The net cooling capacity of the refrigerator is calculated by the following relation:

$$
Q_{\text {net }}=Q_{\max }-Q_{\text {shu }}-Q_{\text {cond }}-Q_{\text {enth }}-W_{\text {ir }}
$$

The gas leakage loss at the clearance between the displacer and the cylinder wall is related to the seal design, manufacturing process, and material used. It cannot be estimated accurately and is ignored. But the loss coefficient $C_{\mathrm{d}}$ can be used to reflect this effect to some extent in addition to the displacer frictional loss. However, the gas leakage at the piston is ignored since it is small for a good compressor design.

\section{System design analysis}

The system design analysis of a split-type free-displacer Stirling refrigerator can be carried out using the present linear network model. For simplification, the following assumptions are made in the analysis:

1 Since the gas in the compressor and the connecting tube are assumed to undergo a stationary and isothermal process, the regenerator inlet temperature $T_{\mathrm{ri}}$ approximates 
Stirling refrigerator design using linear network analysis: B.J. Huang and C.W. Lu

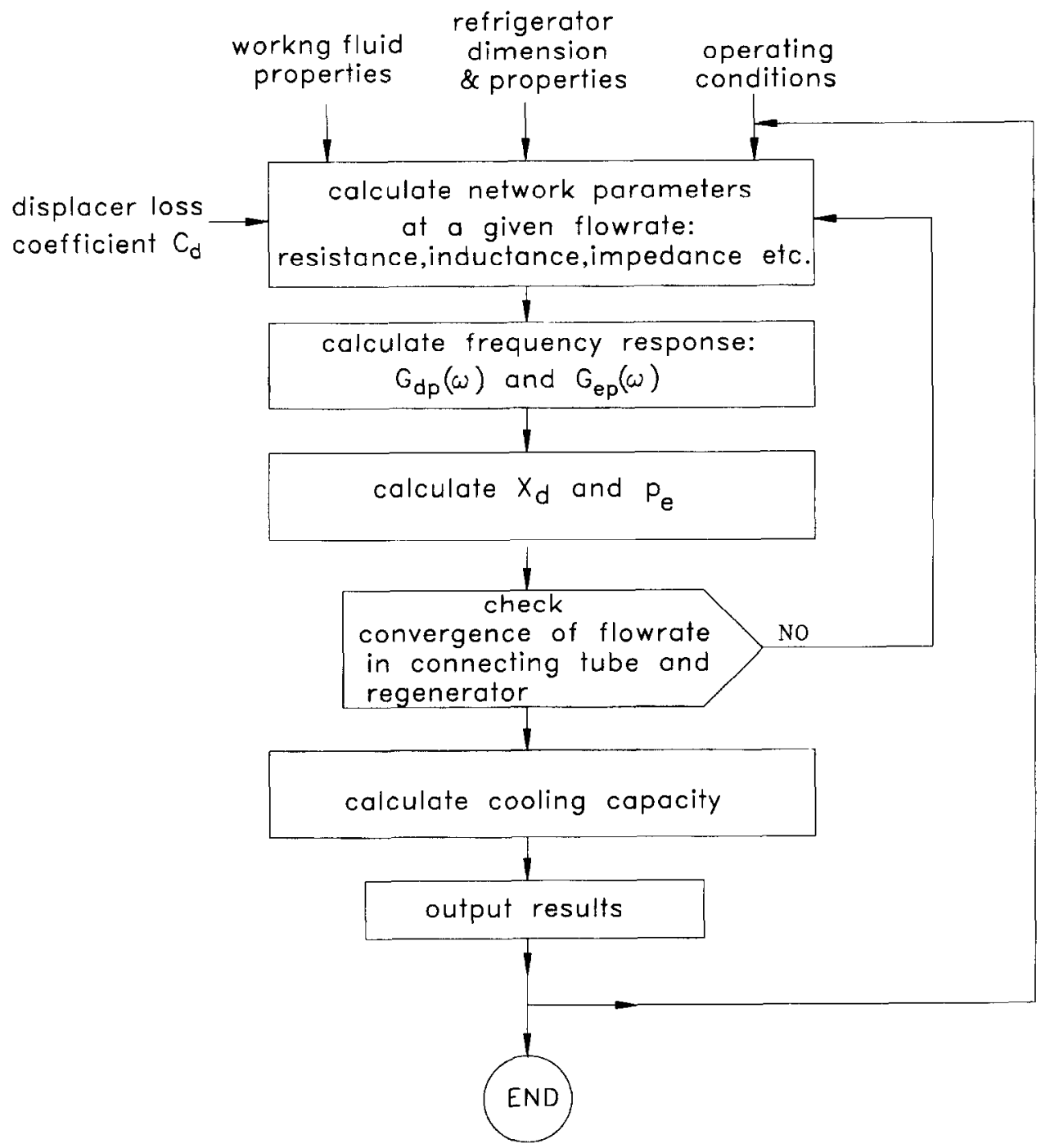

Figure 10 Flow chart of the linear network analysis

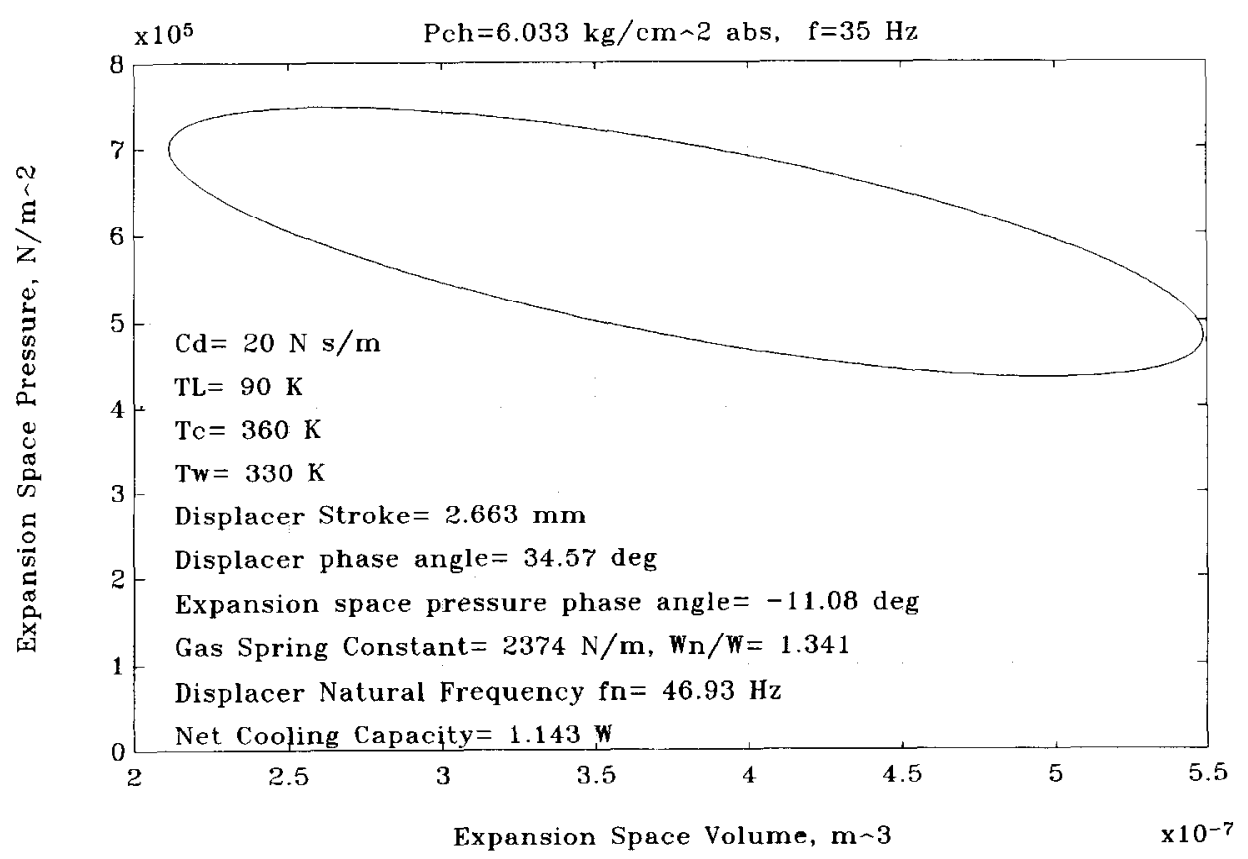

Figure 11 PV diagram of the expansion space 
Stirling refrigerator design using linear network analysis: B.J. Huang and C.W. Lu

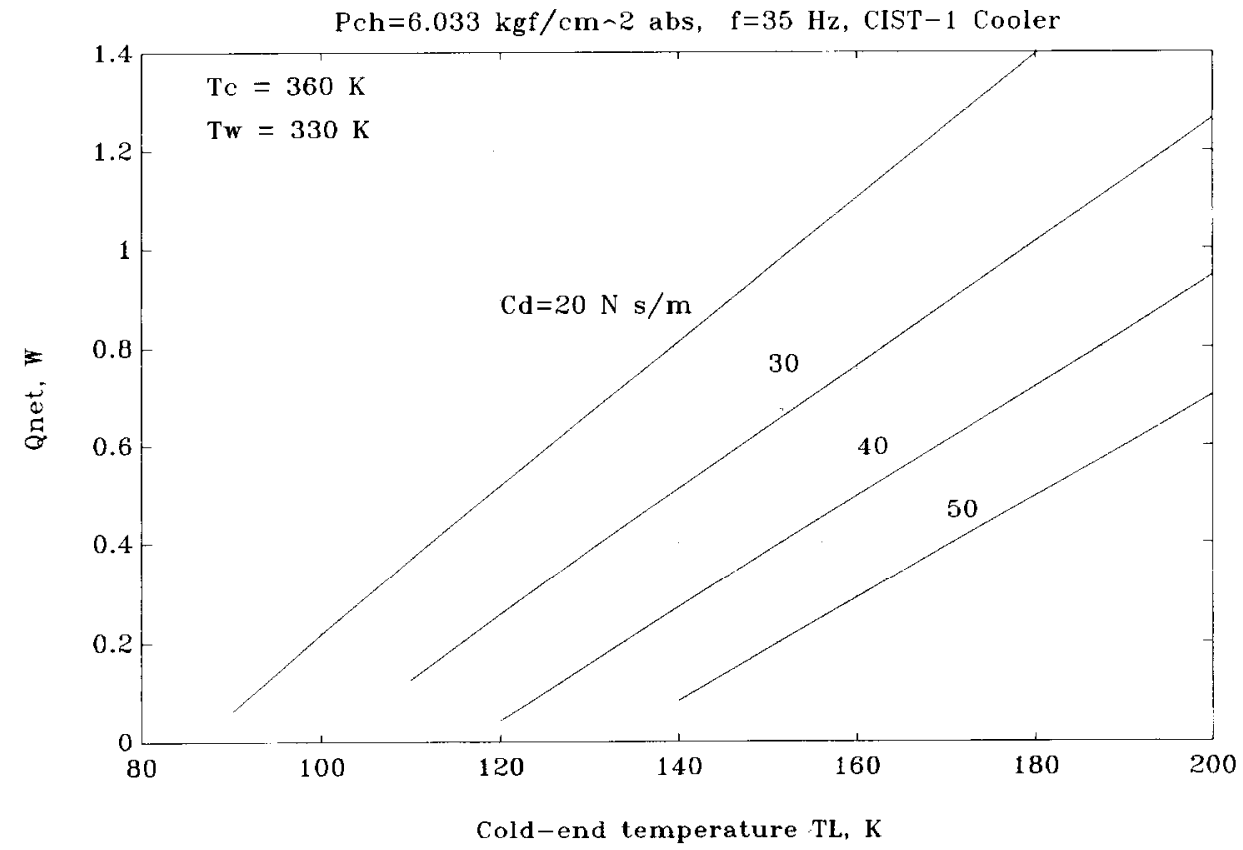

Figure 12 Variation of $Q_{\text {net }}$ with $T_{\mathrm{L}}$

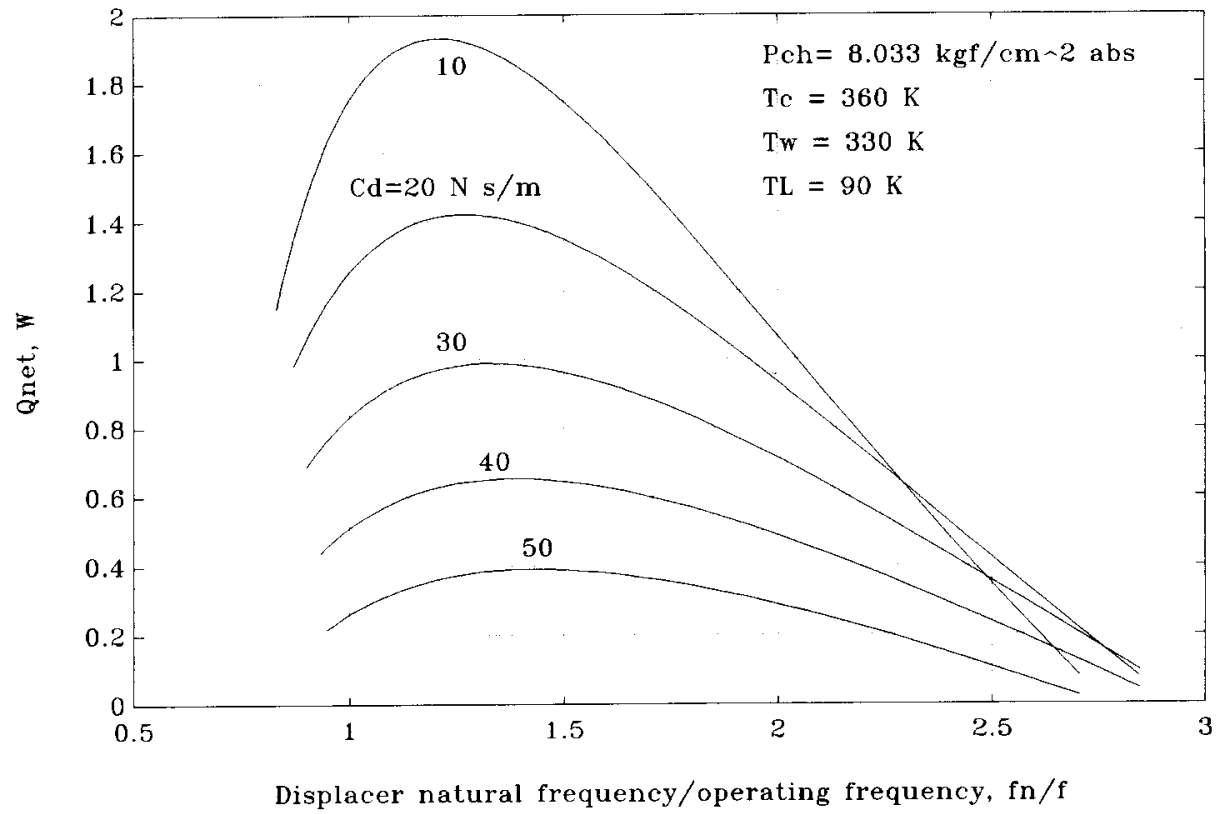

Figure 13 Variation of $Q_{\text {net }}$ with $f$

the compression temperature $T_{\mathrm{c}}$ and the connecting tube temperature $T_{\mathrm{t}}$, i.e.

$$
T_{\mathrm{ri}} \approx T_{\mathrm{t}} \approx T_{\mathrm{c}}
$$

2 The structure of the warm space provides a high thermal inertia to the gas temperature. The gas tempcrature of the warm space was thus assumed constant and equal to the outside wall surface temperature $T_{\mathrm{H}}$ and the regenerator inlet temperature $T_{\mathrm{ri}}$. That is,

$$
T_{\mathrm{w}} \approx T_{\mathrm{ri}} \approx T_{\mathrm{H}}
$$

3 The gas temperature in the cold space $T_{\text {ce }}$ is approximately equal to the outside wall surface temperature of the cold end, $T_{\mathrm{L}}$. This was verified experimentally ${ }^{7}$ since the convective heat transfer between the gas and the wall in the cold space is very large, especially for low $T_{\mathrm{L}}$ and high operating frequency. That is,

$$
T_{\mathrm{ce}} \approx T_{\mathrm{L}}
$$

$T_{\mathrm{L}}$ is assumed to be stationary since the thermal mass of the cold-end exchanger is large as compared to that of the gas inside the cold space.

4 The mean pressure $\bar{P}$ is assumed to be equal to the charge pressure $P_{\mathrm{ch}}$.

5 Since the tube wall usually has a large thermal mass with high thermal inertia, the wall temperatures at the two ends of the regenerator, $T_{\mathrm{ri}}$ and $T_{\text {ro }}$, are approximately constant or stationary and obey the following relations: 


$$
T_{\mathrm{w}} \approx T_{\mathrm{ri}} \approx T_{\mathrm{t}} \approx T_{\mathrm{c}} ; T_{\mathrm{ro}} \approx T_{\mathrm{ce}} \approx T_{\mathrm{L}} \text {. }
$$

Given the refrigerator dimensions, the material physical properties, the operating conditions $\left(T_{\mathrm{L}}, P_{\mathrm{ch}} f, T_{\mathrm{c}}, T_{\mathrm{w}}\right)$, the working fluid properties, and the value of $C_{\mathrm{d}}$, we can carry out system performance analysis according to the flow chart in Figure 10. The present design calculations are carried out for a test refrigerator with design specifications listed in Table 1 . Shown in Figure 11 is the PV diagram of the expansion space. The variation of net cooling capacity $Q_{\text {net }}$ with the cold-end temperature $T_{\mathrm{L}}$ is shown in Figure 12 . $Q_{\text {net }}$ is shown to increase with increasing $T_{\mathrm{L}}$ and decreasing $C_{\mathrm{d}}$. The design calculation is also performed to investigate the variation of $Q_{\text {net }}$ with the operating frequency $f(=$ $\omega / 2 \pi)$. It is shown from Figure 13 that, for a fixed $C_{\mathrm{d}}$ $Q_{\text {net }}$ first increases with increasing $\omega$ (or decreasing $\omega_{n} / \omega$ ), reaches a peak value, and then decreases. An optimum $Q_{\text {ute }}$ is obtained at a frequency ratio $\omega_{n} / \omega$ around 1.2 to 1.5 , depending on the value of $C_{\mathrm{d}}$. That is, the corresponding $\omega$ for the optimum $Q_{\text {net }}$ ranges from 0.67 to $0.83 \omega_{n}$. This coincides with the test results ${ }^{8,9}$.

The frequency response functions of the refrigerator, $G_{\mathrm{dp}}(j \omega)$ and $G_{\mathrm{ep}}(j \omega)$, can also be calculated. The results are shown in Figures 14 and 15 . The amplitude and the phase of the displacer motion are shown to decrease with increasing operating frequency as shown in Figure 14. However, there is a lower limit ( $20 \mathrm{~Hz}$ for the simulated case) for the operating frequency below which $Q_{\text {net }}$ will become negative (i.e. the refrigerator has no cooling capacity). For the gas pressure in the expansion space, the amplitude first increases with increasing frequency, reaches a maximum value then declines. An optimum amplitude exists $(60 \mathrm{~Hz}$ in the simulated case). Though the maximum available cooling capacity $Q_{\max }$ is proportional to the integral of $p_{\mathrm{e}}$ and $X_{\mathrm{d}}$ (i.e. the enclosed area in the PV diagram shown in Figure 11 ), the operating frequency for the optimum $p_{\mathrm{c}}$ may not result in an optimum $Q_{\text {net }}$. This is caused by the variation of the phase angle between $p_{\mathrm{e}}$ and $X_{\mathrm{d}}$.

Table 1 Design specifications of a test refrigerator

\begin{tabular}{|c|c|}
\hline $\begin{array}{l}\text { 1. Compressor } \\
\text { piston diameter } \\
\text { piston stroke } \\
\text { swept volume }\end{array}$ & $\begin{array}{l}26 \mathrm{~mm} \\
12 \mathrm{~mm} \\
6.4 \text { c.c. }\end{array}$ \\
\hline $\begin{array}{l}\text { 2. Connecting tube } \\
\text { length } \\
\text { inside diameter }\end{array}$ & $\begin{array}{l}190 \mathrm{~mm} \\
1.76 \mathrm{~mm}\end{array}$ \\
\hline $\begin{array}{l}\text { 3. Warm space } \\
\text { volume }\end{array}$ & 1 c.c. \\
\hline $\begin{array}{l}\text { 4. Gas spring chamber } \\
\text { plunger diameter }\end{array}$ & $8 \mathrm{~mm}$ \\
\hline $\begin{array}{l}\text { 5. Regenerator } \\
\text { wire screen material } \\
\text { wire screen mesh no. } \\
\text { wire screen length } \\
\text { wire screen diameter } \\
\text { number of screen disks }\end{array}$ & $\begin{array}{l}\text { SS316 } \\
200 \\
60 \mathrm{~mm} \\
11 \mathrm{~mm} \\
880\end{array}$ \\
\hline $\begin{array}{l}\text { 6. Displacer } \\
\text { total mass } \\
\text { rod diameter }\end{array}$ & $\begin{array}{l}27.3 \mathrm{~g} \\
12.7 \mathrm{~mm}\end{array}$ \\
\hline $\begin{array}{l}\text { 7. Expansion space } \\
\text { diameter }\end{array}$ & $12.7 \mathrm{~mm}$ \\
\hline
\end{tabular}

\section{Modification of linear network analysis}

\section{Experimental verification of linear network analysis}

For verifying the linear network analysis, a test refrigerator was built in the present study. The design specifications are shown in Table 1 . Helium gas with $99.999 \%$ purity was used as the working fluid. An electrical heating strip was taped on the surface of the cold head as the cooling load. The cold finger was put in a vacuum chamber for the measurement of net cooling capacity. The test results are shown in Figure 16.

The deviation between the simulation results with $C_{\mathrm{d}}=$ $20 \mathrm{~N} \mathrm{~m} / \mathrm{s}$ using the linear network analysis and the test results are small for $T_{\mathrm{L}}<105 \mathrm{~K}$, but large (underestimated $Q_{\text {net }}$ ) for $T_{\mathrm{L}}>105 \mathrm{~K}$. Figure 16 shows that the present linear network analysis with a constant $C_{\mathrm{d}}$ is satisfactory at low $T_{\mathrm{L}}$. However, it will not be guaranteed all the time in selecting the value of $C_{\mathrm{d}}$, since $C_{\mathrm{d}}$ depends on the displacer seal design, the manufacturing quality and the material used. There is no way for a designer to predict $C_{\mathrm{d}}$ accurately without field experience. It may also not be true to assume a constant value of $C_{\mathrm{d}}$ for various operating conditions.

\section{Modification of linear network analysis}

In order to improve accuracy in performance prediction, a modification of the linear network analysis is necessary. In the present analysis, a loss coefficient of the displacer $C_{\mathrm{d}}$ is included. $C_{\mathrm{d}}$ is the coefficient accounting for the friction and gas leakage losses of the displacer at the surface in contact with the cylinder of the cold finger and at the clearance seal of the gas spring. Hence, $C_{d}$ may not be constant but can vary with the displacer oscillating frequency $f$ and cold-head temperature $T_{\mathrm{L}}$.

In the present study, the value of $C_{\mathrm{d}}$ at various operating conditions is determined by fitting the test results of $Q_{\text {net }}$ with the calculated value. An empirical correlation of $C_{\mathrm{d}}$ is obtained from the analysis of more than 200 test data, with $T_{\mathrm{L}}$ in $K$ and $f$ in $\mathrm{Hz}$

$$
\begin{aligned}
C_{\mathrm{d}}\left(f, T_{\mathrm{L}}\right)= & \left(430.66+\frac{1.0851}{T_{\mathrm{L}}}+\frac{7.7346 \times 10^{6}}{T_{\mathrm{L}}^{2}}\right) \\
& \left(0.6653+0.2604 f-5.212 \times 10^{-4} f^{2}\right)
\end{aligned}
$$

The analytical results using the above empirical relation coincide with the test results of $Q_{\text {net }}$ to within $\pm 10 \%$, as shown in Figure 16. $C_{\mathrm{d}}$ obtained from the experiments includes the effect of gas leakage in addition to the frictional loss.

\section{Discussion and conclusion}

A linear network model is developed for the system performance analysis of split-type free-displacer Stirling refrigerators. The dynamics models are derived to describe the input/output relation of each component by use of the governing equations in conjunction with linearization and approximation. By connecting the equivalent circuits of the components together, a linear network consisting of a fluid network and a displacer network is obtained. Two transfer functions are derived for the displacer motion and the gas 
Stirling refrigerator design using linear network analysis: B.J. Huang and C.W. Lu
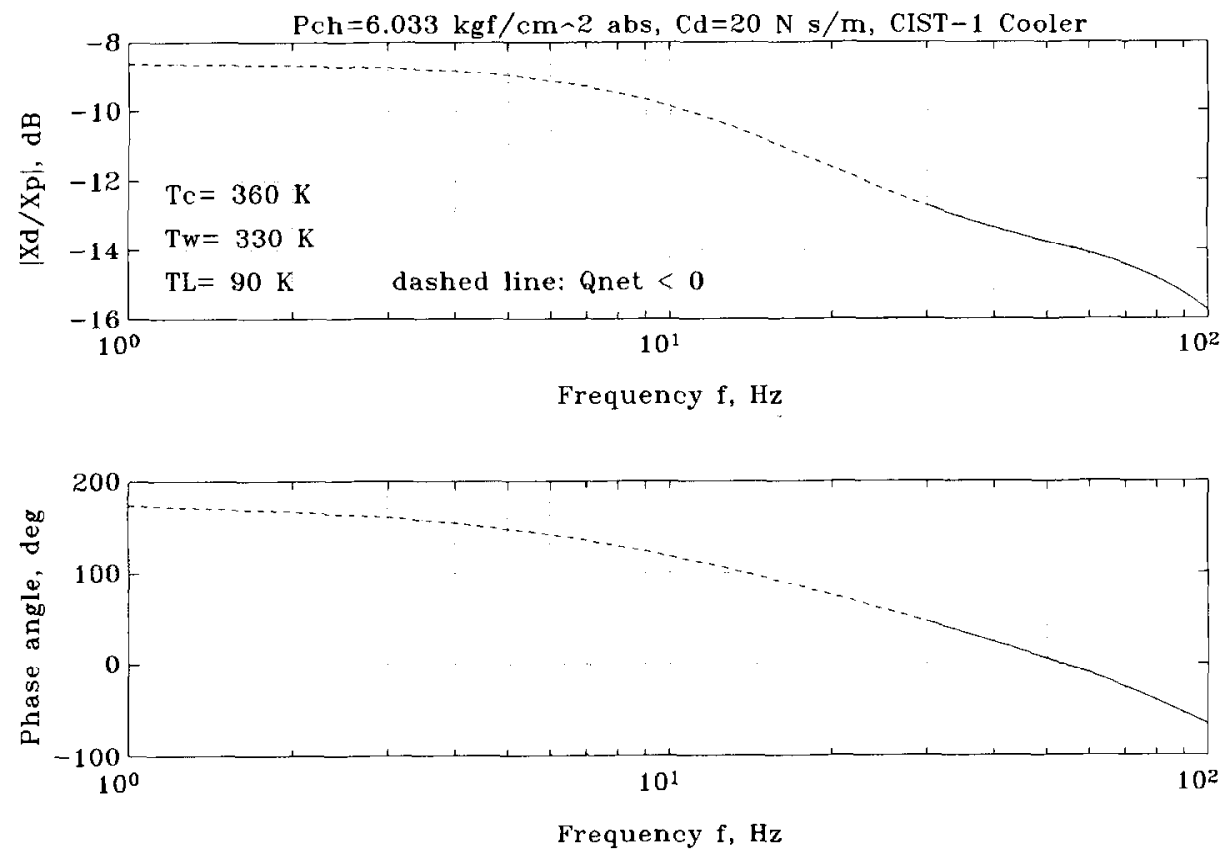

Figure 14 Frequency response of $G_{\mathrm{dp}}(s)$
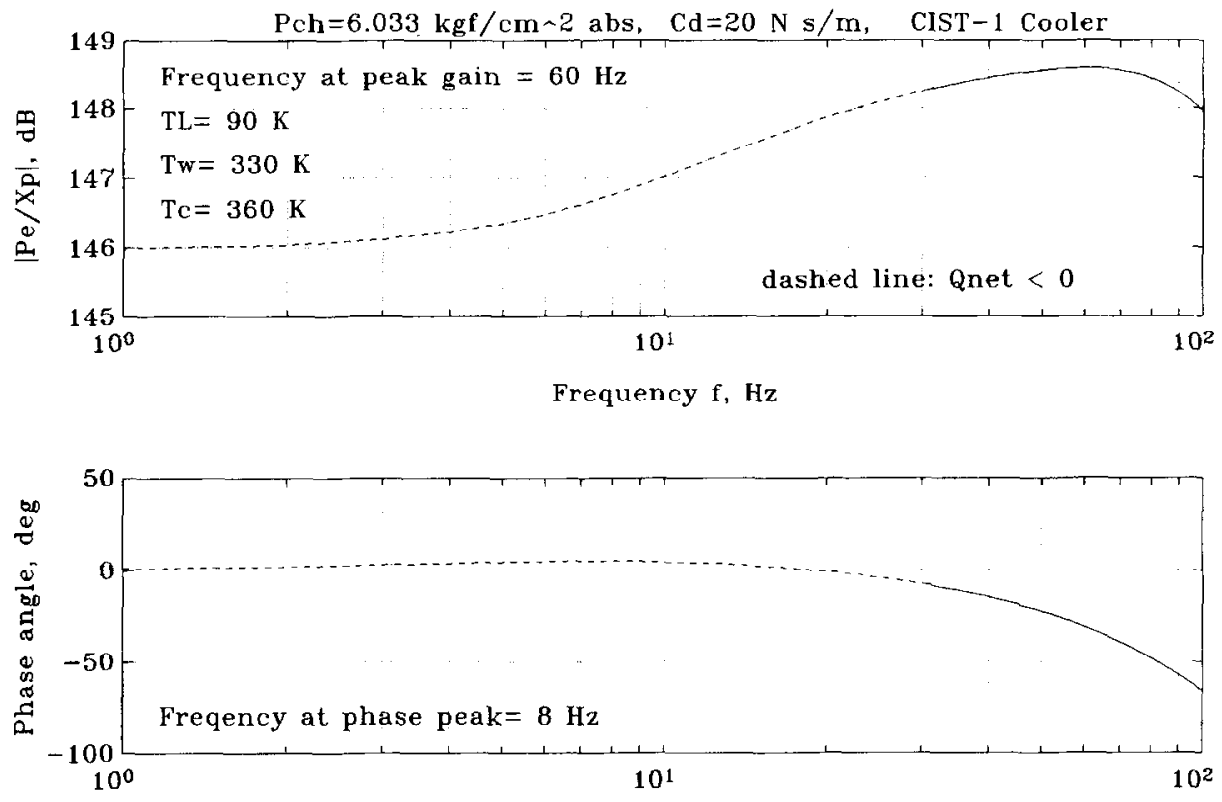

Frequency $\mathrm{f}, \mathrm{Hz}$

Figure 15 Frequency response of $G_{\mathrm{ep}}(s)$

pressure of the expansion space. The system performance can then be evaluated from the frequency response of the two transfer functions by using the sinusoidal signal analysis. Since the analytical solutions are obtained, the computation takes only a few seconds on a PC for each design calculation.

Implementation of the linear network model in the system analysis of a split-type free-displacer Stirling refrigerator relies on the correct selection of $C_{\mathrm{d}}$ value. $C_{\mathrm{d}}$, however, is determined mainly from experience. The value of $C_{\mathrm{d}}$ has been shown in the present study to vary with the operating frequency $f$ and the cold-end temperature $T_{\mathrm{L}}$. An empirical correlation of $C_{\mathrm{d}}$ is obtained. The performance prediction of a Stirling refrigerator using the empirical relation of $C_{\mathrm{d}}$ is satisfactory. The $C_{d}$ empirical relation can be further updated if more test results of various hardwares are available. The present analysis is shown to be superior to the other methods in its high computation speed and good accuracy. It is now possible for us to develop a powerful CAD tool for the design of split-type free-displacer Stirling refrigerators using the linear network model.

\section{Acknowledgement}

The present study was supported by the National Science Council, Taiwan, through Grant No. NSC81-0401-E002587, No. CS83-0210-D-002-011 and No.CS84-0210-D002-027. 


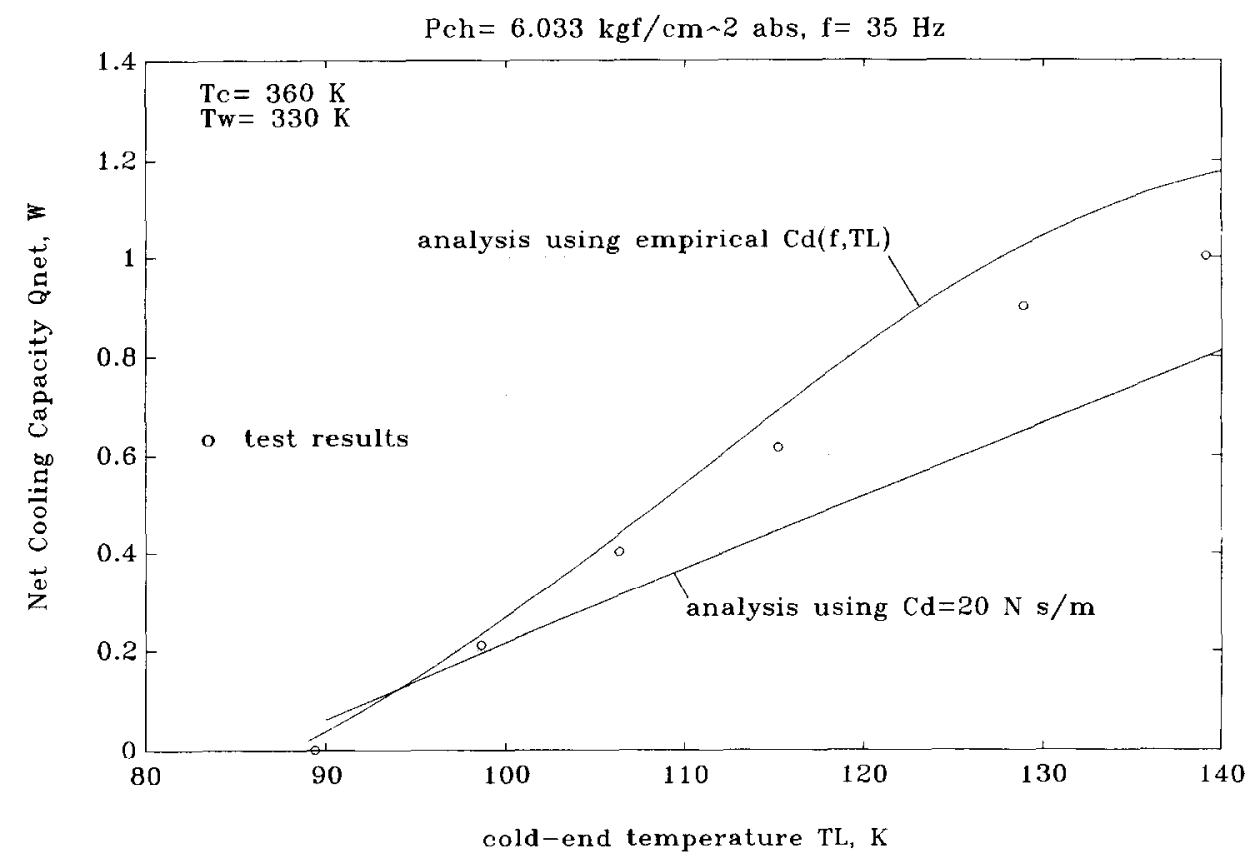

Figure 16 Comparison of the test results with the analysis

\section{References}

1 Yuan, S.W.K., Spradley, I.E., Yang, P.M. and Nast, T.C. Computer simulation model for Lucas Stirling refrigerators Cryogenics (1992) $32 \quad 143-148$

2 Huang, B.J. and Lu, C.W. Linear network analysis of split-type Stirling refrigerator Cryogenics 34 ICEC Supplement (1994) 34 207-210

3 Lo, T.C. Fluid Network Theory Mechanical Engineering Pub Inc, Beijing, 1988 (in Chinese)

4 Huang, B.J. and Lu, C.W. Linear network analysis of regenerator in a cyclic-flow system Cryogenics (1995) 35 203-207
5 Tanaka, M., Yamashita, I. and Chisaka, F. Flow and Heat Transfer Characteristics of Stirling Engine Regenerator in an Oscillating Flow JSME International J, Series 2 (1990) 33 283-289

6 Urieli, I. and Berchowitz, D.M. Stirling Cycle Engine Analysis Adarn Hilber Lid, Bristol, 1984

7 Huang, B.J. and Chang, S.C. System performance analysis of Gifford-McMahon cooler Cryogenics (1995) 35 117-125

8 de Jonge, A.K. A small free piston Stirling refrigerator Proc 14th ICECE (1979) 1136-1141

9 Tailor, P.R. and Narayankhedkar, K.G. Analysis and performance prediction of electro-magnetically-driven free displacer Stirling cryocooler Cryogenics (1988) 28 169-176 